Journal of Engineering and Applied Sciences 15 (3): 826-829, 2020

ISSN: 1816-949X

(c) Medwell Journals, 2020

\title{
A Review of Accelerometer Sensor and Gyroscope Sensor in IMU Sensors on Motion Capture
}

\author{
Ilham Arun Faisal, Tito Waluyo Purboyo and Anton Siswo Raharjo Ansori \\ Department of Computer Engineering, Faculty of Electrical Engineering, \\ Telkom University, Bandung, Indonesia
}

\begin{abstract}
IMU is electronic devices that used on specific body, angular rate and sometimes magnetic fields surround the body, use combination of accelerometer sensor and gyroscopes sensor, sometimes magnetometer to but IMU usually just using accelerometer and gyroscope sensor. IMU Sensor usually used on aircraft maneuver include Unmanned Aerial Vehicles (UAV) and space aircraft include landers and it's satellite. Accelerometer is an automatic tool for measuring acceleration, detecting and measuring vibration (vibration) and measuring acceleration due to the body (inclination). The accelerometer can be used to measure vibrations in cars, engines, buildings and security installations. Gyroscopes is device that mounted to a frame and can sensing an velocity of angular if that frame is rotate. There is some gyroscopes classes, depending on the operation physical and technology that involve.
\end{abstract}

Key words: IMU, accelerometer sensor, gyroscope sensor, vibration, velocity, physical

\section{INTRODUCTION}

IMU sensors usually has accelerometer sensor and gyroscopes sensor (Filippeschi et al., 2017). A common sensing approach used for accelerometer are sense capacitance that velocity is relate to replace in a move mass capacitance (Dadafshar, 2014). Gyroscopes are devices that used on frame and can sense the speed of angular velocity if frame is rotating.

Accelerometer Sensor is used for sense capacitance that accelerations are relate for changing a capacitance of a shifting mass. Gyroscope sensor and accelerometer sensor have advantages and disadvantages of each. The slope angle calculated from the accelerometer data has a slow response time while the integrated slope angle of the gyroscope data has a deviation over a certain period of time. It can be said that the data on the accelerometer is useful for the long term while the gyroscope is used for the short term. One of the simplest ways to combine data from a gyroscope and accelerometer is to use complementary filter.

\section{MATERIALS AND METHODS}

In this study review will discuss about IMU sensors which use on motion capture. IMU sensors usually has accelerometer and gyroscopes sensor. This signals has a different function depending on the application to system. Usually, IMU is used for pose reconstruction or change a position of body oriented that sensor are attached. IMU are used for signal sensor integration over time to estimate speed, position and oriented. Because the two sensor, accelerometer sensor and gyroscopes Sensor suffer biases and sound variation, this oncoming cause to a early estimation which unreliable after a few seconds. But researcher starting investigate the algorithm and hardware to solve shift problems. Many cases, IMU are using three axis magnetometer. The magnetometer measures magnetic fields (Earth) that used for estimating reference on earth-fixed from IMU orientation (Filippeschi et al., 2017).

Typically rate-gyroscopes are three orthogonal and accelerometers that are measure an angular velocity and acceleration linear in a three dimensional space are contained in an IMU. In relation to starting point, velocity and orientation, accelerometers and gyroscopes are used to tracking a position and orientation of object. Recent advances in MEMS technology have made it possible to keep tracking of human motion and orientation of devices with the possibility to manufacture smaller and lighter sensors (Dadafshar, 2014).

Accelerometer sensor: Accelerometer is an automatic tool for measuring acceleration, detecting and measuring vibration (vibration) and measuring acceleration due to the body (inclination). The accelerometer can be used to measure vibrations in cars, engines, buildings and security installations. An accelerometer can also be applied to measuring electronic equipment, like 3-dimensional games, computer mice and telephones and an earthquake activity and can be used for multimedia purposes like VOD (Video on Demand) which that video use a 3D 
motion and the 3D object can be transform into image like JPG which has continuous function from light intensity in a dimension (Selany et al., 2015; Sugara et al., 2018). For more advanced applications, there are many sensors for navigation purposes. Acceleration create a state on speed along the time. Increase the speed on range time called acceleration. But if speed decreased from the previous speed, it's called deceleration. Acceleration also depending on direction or orientation because it's a decreased velocity on vector quality. Direction movement are changing of objects will also cause acceleration. To buy distance data from the accelerometer sensor, a dual integral process is required for the output sensor . One of the accelerometer sensor applications that is currently being developed is to detect motion such as foot movements as a navigation function and also movement as a console game or for other controls.

Basic principle of accelerometer: For simplicity, MEMS accelerometer in one dimension can basically be seen as a process operating of Newton's second law where a mass is attached to a spring within a reference frame (Fig. 1).

There are two main principles of MEMS accelerometers where one is to measure the displacement of the mass and the other one measures frequency of a vibrating element are changing (mass) it's caused because of tension changing. The accelerometer measures linear acceleration and by integrate the signal twice we can obtain the position (Grahn, 2017).

Capacitive bandwidth just a slight hundred hertz by the physical geometry (spring) and air trap within IC which act like a reducer (Dadafshar, 2014):

$$
\mathrm{c}=\frac{\varepsilon^{\times} \times \varepsilon^{\times} \mathrm{a}}{\mathrm{d} \times \text { Farrad }}
$$

$\varepsilon_{0} \quad:$ Empty room that permit to

$\varepsilon_{\mathrm{r}} \quad$ : Materials among plate

a : Wide over lap among electrode

đ : Splitting among electrode

Farrad : SI derived unit

When you choose an accelerometer for application, you have to consider about some characteristic as (Fig. 2):

Bandwidth (Hz): Sensor for badwidth are indicates the range about vibration of frequency that responded by accelerometer or sometimes the best reading can be done. Human can't build body movement past the range from $10 \mathrm{~Hz}$. So, bandwidth range $40-60 \mathrm{~Hz}$ are enough for sense a tilt or people movement.

Sensitivity ( $\left.\mathbf{L S B g}^{-\mathbf{1}}, \mathbf{m V g ^ { - 1 }}\right)$ : Sensitivity have a function to quantify of a below signal that can be

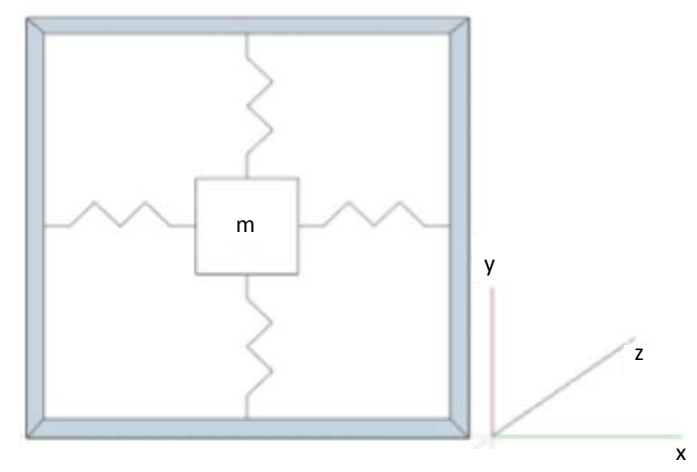

Fig. 1: Illustration of the basic principle of Newton's second law in two dimensions where a mass $\mathrm{m}$ is attached to springs within a reference frame (Grahn, 2017)

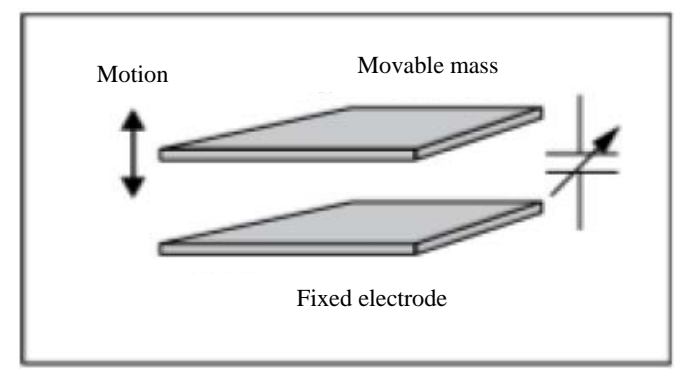

Fig. 2: Capacitance And mass moving (Dadafshar, 2014)

detected or change an electric signal output per change in input electric signal input. It's valid for a frequency.

Voltage noise solidity ( $\mu$ g/SQRT Hz): It's change with square root that are inverse of bandwidth. The quicker we read the change of accelerometer, the bad we can obtain. Noise have bigger influence for accelerometer performance.

Zero-G voltage: It's term specified range of frequency which can be expect are output below $0 \mathrm{~g}$ accelerations.

Response frequency $\mathbf{( H z ) : ~ T h i s ~ r a n g e ~ t h a t ~ s p e c i f i e d ~ b y ~}$ band toleration ( $\pm 5 \%$, etc.) that sensor will get a motion and tell the right outcome. A band toleration let's the consumer counts how many sensitivity of devices from frequency references sensitivity at any frequency range.

Dynamic range (g): Dynamic Range are range of amplitude which smallest detectable that can measure by accelerometer to biggest amplitude before distortion or clipped outcome of signal (Dadafshar, 2014). 
Table 1: Resume sensor for IMU sensor, accelerometer sensor and gyroscopes sensor

\begin{tabular}{|c|c|c|}
\hline Materials & Author's name & Discussion \\
\hline IMU sensor & Filippeschi et al. (2017) & $\begin{array}{l}\text { IMU sensors usually has accelerometer Sensor and gyroscopes } \\
\text { sensor }\end{array}$ \\
\hline \multirow[t]{4}{*}{ Accelerometer sensor } & $\begin{array}{l}\text { Muhammad Riyadi, Wahyudi, Iwan } \\
\text { Setiawan (Muhammad Riyadi in 2010) }\end{array}$ & $\begin{array}{l}\text { Accelerometer is an automatic tool for measuring acceleration, } \\
\text { detecting and measuring vibration and measuring acceleration due } \\
\text { to the body }\end{array}$ \\
\hline & Dadafshar (2014) & $\begin{array}{l}\text { The sensing oncoming used to accelerometer the capacitance } \\
\text { where velocity is relate to changes in mobility of the move mass }\end{array}$ \\
\hline & Grahn (2017) & $\begin{array}{l}\text { MEMS accelerometer in one dimension is where a mass is attached } \\
\text { to a spring within a reference frame }\end{array}$ \\
\hline & $\begin{array}{l}\text { (Sudirman Hamonangan } \\
\text { Sihombing in 2012) }\end{array}$ & $\begin{array}{l}\text { One of the accelerometer sensor applications that is currently being } \\
\text { developed is to detect motion }\end{array}$ \\
\hline \multirow[t]{2}{*}{ Gyroscopes sensor } & $\begin{array}{l}\text { Sugara et al. (2018) and } \\
\text { Passaro et al. (2017) }\end{array}$ & $\begin{array}{l}\text { Gyroscopes is device that mounted to a frame and can sensing } \\
\text { velocity of angular if it's frame is rotate }\end{array}$ \\
\hline & Grahn (2017) & $\begin{array}{l}\text { MEMS gyroscope make use of experience that called Coriois } \\
\text { effect which declare that in a frame of reference rotating at angular } \\
\text { velocity, a m mass is move with velocity }\end{array}$ \\
\hline
\end{tabular}

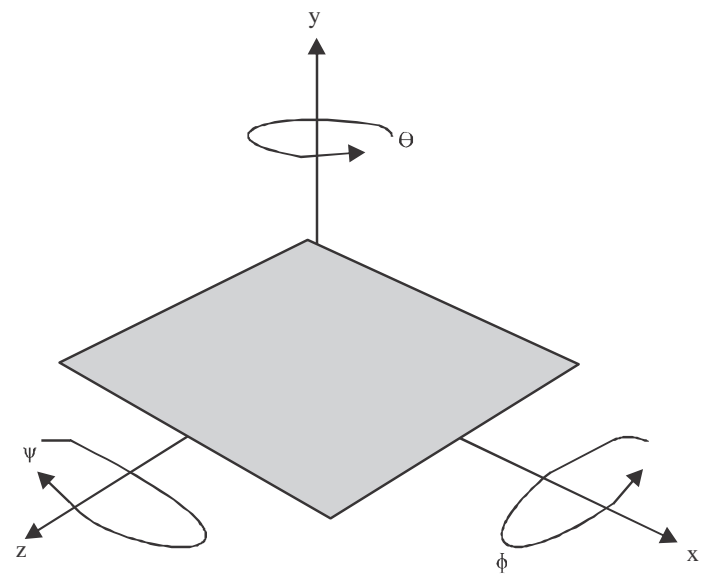

Fig. 3: Illustration of the basic principle of a gyroscope and how the rotations $(\Phi, \theta, \Psi)$ occur around respective axes (Grahn, 2017)

Gyroscopes sensor: Gyroscopes is device that mounted to a frame and can sensing velocity of angular if it's frame is rotate. There is some gyroscopes classes, depend on the operation physical and technology that involve. Gyroscope can be standalone or used for something complex system, like Inertial Measurement Unit (IMU), gyrocompass, attitude heading reference system and navigation system (Passaro et al., 2017) (Fig. 3) (Table 1).

\section{RESULTS AND DISCUSSION}

Basic principle of gyroscope: MEMS gyroscope make use of experience that called Coriois effect which declare that in a frame of reference rotating at angular velocity, a $\mathrm{m}$ mass is move with velocity! experience a force (Grahn, 2017):

$$
\mathrm{Fc}=-2 * \mathrm{~m}\left(\omega^{*} \mathrm{v}\right)
$$

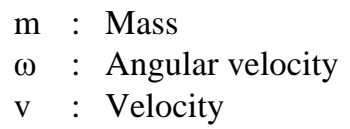

To measure the Coriolis effect, MEMS gyroscope contains vibrating masses which vibrates along a drive axis. A secondary vibration is induced with the perpendicular sense axis that displaces the mass from it's original path when the gyroscope is rotated (Grahn, 2017). The working principles of a gyroscope where the rotation around respective axes. The gyroscope introduces capacitance changes to detect this displacements. Based on this, the angular velocity of the IMU can be measured and by integrate the signal, we can obtain orientation (Passaro et al., 2017).

Mechanical gyroscopes basically consist of spin mass which rotate around it's axis. Particularly, when the mass are rotating on it's axis, it's tend store main parallel for itself and cancel any attempt to change the oriented. If gyroscopes are discharged on gimbals which permit mass for navigating in three direction space, axis would remain orienting on the equal direction, in fact the directions is change. Mechanical gyroscopes show several physic phenomena include precession and nutation (Passaro et al., 2017).

\section{CONCLUSION}

In this study, we have review about accelerometer sensor and gyroscopes sensor on IMU sensors. The two sensors that used for IMU sensors is accelerometer and gyroscope, accelerometer is sensing capacitance that acceleration are related to changing the capacitance of a shifting mass, then gyroscopes sensors is for sensing an angular velocity if frame is rotate. 


\section{REFERENCES}

Dadafshar, M., 2014. Accelerometer and gyroscopes sensors: Operation, sensing and applications. Maxim Integrated, Vol. 1,

Filippeschi, A., N. Schmitz, M. Miezal, G. Bleser, E. Ruffaldi and D. Stricker, 2017. Survey of motion tracking methods based on inertial sensors: A focus on upper limb human motion. Sens., Vol. 17, No. 6. 10.3390/s17061257.

Grahn, E., 2017. Evaluation of MEMS accelerometer and gyroscope for orientation tracking nutrunner functionality. KTH, School of Technology and Health, Huddinge, Sweden. http://www.divaportal.org/smash/record.jsf?pid=diva2\%3A1146723 \&dswid=-2386
Passaro, V.M.N., A. Cuccovillo, L. Vaiani, M. De Carlo and C.E. Campanella, 2017. Gyroscope technology and applications: A review in the industrial perspective. Sens., Vol. 17, No. 10. 10.3390/s17102284

Selany, S., S.M. Nasution and T.W. Purboyo, 2015. Sosemanuk algorithm for encryption and decryption Video on Demand (VoD). Proceedings of the 2015 IEEE Asia Pacific Conference on Wireless and Mobile (APWiMob'15), August 27-29, 2015, IEEE, Bandung, Indonesia, pp: 125-129.

Sugara, N.L., T.W. Purboyo and A.L. Prasasti, 2018. Comparative analysis of image compression using huffman and DCT Method on JPG Image. J. Eng. Applied Sci., 13: 4447-4452. 